\title{
Creación poética en la Generación de 1987. ¿Qué queda de una escena en conflicto ${ }^{1}$
}

\author{
Thomas Harris² \\ principe.prospero@gmail.com
}

\section{Introducción}

Creo que si nos internamos en los aspectos más significativos de la llamada nueva escena (ya remota) de la poesía de los años ochenta o de la generación de 1987, como prefiere denominarla el poeta y miembro de esta misma generación, Andrés Morales, destacaría algunos poetas o núcleos de poetas y sistemas de preferencias líricas, que, por una parte, apelan a la continuidad con la tradición precedente (el mismo Morales, Eduardo Llanos, Jorge Montealegre, entre otros): la poesía escrita por mujeres, o de género, o femenina, como se la quiera llamar, que per se transgresora y fundacional, en la medida en que debe, tiene, es impelida y se auto-exige construir una nueva escena, tanto a nivel discursivo como temático -porque se habla desde y sobre la mujer- (poesía desplazada por un discurso digamos patriarcal preponderante desde los comienzos de nuestra lírica: cabe recordar que las poetas de la generación del 50 fueron, de alguna manera, "rescatadas" y resignificadas por la generación de 1987, y que en la del 60 solo destacó un nombre: Cecilia Vicuña, con una poética muy particular, performática, chamánica, ritual, etcétera) y que la toma del campo por la mujer y su escritura, en esta generación anómala, cubre un momento de aparición y vigencia en poetas nacidas que abarcan desde la década del 40 -Eugenia Brito, Soledad Fariña, Alejandra Basualto-, entre otras, a comienzos del 60 -Bárbaro Délano y Rosabbety Muñoz-); la poesía etnocultural, como la Ilamó Iván Carrasco, y que creo que ahora, por una parte, coincide en la escena geopoética del Sur de Chile o la llamada, por sus miembros, Suralidad -Riedemman, Mansilla, Rosabetty Muñoz, Juan Pablo Riveros entre los más destacados- y la poesía de nuestros pueblos originarios, que surgió como una reivindicación sobre todo del mapundungun como portador de una oralidad literaria, y que sin duda inaugura y sitúa en la escena Elicura Chihuailaf, y, también un poco más tarde Graciela Huinao, y cuyas pertinencias y reivindicaciones eran doblemente políticas, puesto -y aún esto continúa con las nuevas generaciones: Jaime Luis Huenún, Rosana Miranda Ruipailaf- que debían confrontarse con la dictadura militar y, a la vez, el huinca, nosotros, los chilenos.

Finalmente, donde centraré este texto, será lo que denominaré -ciñéndome a la nomenclatura utilizada, entre otros críticos, por Oscar Galindo, Marcelo Rioseco, Eugenia Brito, Jaime Concha, etcétera, como neovanguardia,

\footnotetext{
1 Texto leído al fin de la estancia del autor en el programa "Escritor en Residencia" en la Pontificia Universidad Católica Chile, primer semestre de 2016.

2 Poeta, Jefe de Ediciones de la Biblioteca Nacional, Secretario de Redacción de revista "Mapocho".
} 
porque en la época, en la escena de los 80 , hubo una poesía que no se inscribe en las diferencias de género ni culturales, y tampoco apuesta por la continuidad con la tradición -entiéndase esta como el continuum de un canon específico- sino que intenta a través de una revisitación de las vanguardias orgánicas, al decir de Bernardo Subercaseaux, de comienzos del siglo XX, construir otra escena, confrontacional y trasgresora, no solo con la continuidad de la tradición sino también contra la dictadura cívico militar de los 70/80 y cuyos rasgos, pertinencia y estrategias trataremos de especificar en lo que sigue.

\section{La cuestión de la neovanguardia de los años 80}

Pensamos que es pertinente afirmar la existencia de un corpus de poetas que se inscribió en una escena textual -y que dialogó con otra extratextualque podríamos llamar -¿sin equívocos?- neovanguardia, y sus respectivas funciones dentro de esta escena inscrita en el turbulento y traumático campo cultural de los 80 . Pero debemos detenernos un tanto, para comprobar la pertinencia, primero en su génesis, o, para parafrasear a Bernardo Subercaseaux en relación a las vanguardias orgánicas, heroicas o fundacionales de principios del siglo XX, como las ha denominado indistintamente el discurso crítico, su genealogía $y$, además y sobre todo, sus procedimientos textuales. O sea, ya pasados, casi 35 años, ¿quién habló primero de la neovanguardia?; ¿cuál fue su génesis bautistmal?; ¿fue una necesidad textual y vital?; ¿cómo y porqué se desligó o se propuso como una nueva con la tradición que venía dialogando en tanto continuidad hasta 1973 ?

En principio, como en todos estos casos, asoman ciertos indicios en el campo, que se están produciendo giros, algunos desequilibrios, señales que se quieren instalar, tomarse la escena. Tal vez un preámbulo premonitor sea la revista Manuscritos (1975), cuyo comité de redacción estaba conformado por un variopinto y heterogéneo grupo de intelectuales: Felipe Alliende, Antonio Arbea, Joaquín Barceló, Cedomil Goic, Mario Góngora, Jorge Guzman, Alvaro Jara, Enrique Lihn, José Ricardo Morales, Nicanor Parra y Juan de Dios Vial, y editada por el ya mítico y desaparecido Centro de Estudios Humanísticos de la Escuela de Ingeniería de la Universidad de Chile- que ya apela cierto desasosiego e incompostura cultural, al invocar el mítico Quebrantahuesos perpetrado por Enrique Lihn, Nicanor Parra y Alejandro Jodorowsky, y agiornado por un sesudo discurso teórico de Ronald Kay; la publicación de "Areas verdes" de Raúl Zurita (como una suerte de poesía de adelanto de lo más innovador de su libro Pugatorio publicado por Universitaria en 1979); una sección de la revista de textos de Nicanor Parra, con gestos escriturales y gráficos que se quieren más adelantados y con un tic gráfico mayormente vanguardista, que los Artefactos de 1973; El rincón de los niños, una novela/ vida de Cristián Hunneus que nunca se termina como proyecto sino con la desaparición -muere- del autor, y una gráfica ad hoc que, pensamos, ya anuncia la revista CAL (junio de 1979) dirigida por Nelly Richard, quién posteriormente escribe uno de los libros más significativos, para una comprensión situacional del campo cultural de la época de la dictadura militar, Márgenes e Instituciones. El arte en Chile desde 1973 (1986). La revista Cal será el depositario inicial, gráfica, performática, teórica y situacional tanto de la escena de Avanzada como de la neovanguardia. La una y las otras de 
efímera producción y circulación (un número único, Manuscritos, y tres, Cal) sea por los motivos contextuales que sean, rasgos propios de toda publicación vanguardista orgánica o fundacional.

Es en Cal -sobre todo el primer número-, la expresión impresa de la galería de arte homónima, la revista que insta a los colaboradores a participar en un dossier teórico, donde la impronta -y la petición programática- es dar cuenta del "marco teórico" del proceso creativo de cada participante. Es decir, evidenciar con una "caja de herramientas", del porqué de cada praxis. Es allí donde aparece el Mein Kampf de Raúl Zurita que podríamos considerar como el texto que inicia una genealogía que -polémica desde su, -para algunos poetas sobre todo-desdichado título y provocador, para otros; el texto decía así (lo citamos in extenso por su carácter programático e inaugural):

\section{QUÉ ES EL PARAÍSO}

Gentes de Hiroshima:

Trabajadores chilenos: ¿Qué es el Paraíso?

Naciones de la tierra: ¿Qué es el Paraíso?

Desde los lugares de este exilio sudamericano, como uno repetido, te hablo del trabajo de asumir en los límites de nuestra vida la construcción del Paraíso.

Yo soy un hambriento, esto es, uno repetido en el hambre. Yo sufro, esto es, uno repetido en el sufrimiento. Yo tal vez esté condenado, esto es, uno repetido en la condena.

Yo soy un trabajador del arte y moriré, pero el trabajo del que

te hablo no es una preparación para morir, escuchen el latido

de sus corazones.

Yo trabajo en la obra del Paraíso, pero como uno más en el recorrido de su vida. Y trabajar con la vida es trabajar con la corrección sistemática de la propia experiencia como un borrador de la experiencia que será, de la vida que alguna vez

será. Es un proyecto de construcción de un nuevo sentido y de una nueva forma social de experiencia.

Entonces el trabajo en la obra del Paraíso no es solo un trabajo de arte sino de corrección del dolor de la experiencia. Yo persisto en ello, pero no como un escritor o un artista. $O$ al menos no como eso solamente, sino como un obrero de la experiencia; como un obrero que penosamente trata de ir corrigiendo los borradores de su camino en la experiencia. Como uno repetido que trata de impugnar el individualismo y las ganancias ilícitas de los individualistas. El individualismo es la plusvalía que el terror frente a la muerte le saca al terror

frente a la vida sí mira, per me si va tra la perduta gente. Entiendo entonces la obra del Paraíso como una práctica que desde el dolor, es decir, desde el hambre, desde el terror, desde la 
la soledad, transforme la experiencia del dolor en la construcción

colectiva de un nuevo significado. Comprender que se trata de

la vida de todos, es dar por concluidas las peores formas de la

antigüedad para estampar una nueva marca sobre estos páramos

sudamericanos. Esa marca es lo que la antigüedad olvidó de la

belleza y nosotros, estos cabezas negras, afirmamos nuestro derecho a un trabajo en la belleza.

¿Qué es entonces el Paraíso?

El cielo ha sido desde siempre el lugar que hemos ido Ilenando

con las carencias de la vida. Como tantos, despojado, en el año

1975 inicié mi trabajo entendido como una práctica para el Paraíso, no para el cielo vacío. El inicio de su camino se abre con

el acto de haber quemado mi cara porque todavía no era posible

marcar el cielo con el hecho corregido de nuestras vidas, pero en el

documento de esa quemada se relaciona este acto con las estrellas

de la noche. Yo sé (y mis amigos también) que cuando podamos

rediseñar nuestros trabajos y por ende romper con cualquier obligación al servilismo físico o mental, todos -muertos $y$ vivos-

podremos por fin revertir nuestras carencias y por ende corregir

el cielo. Ese es el camino de mi vida, como uno más repetido,

el Inferno, el Purgatorio y el Paradiso del Mein Kampf de Raúl

Zurita (y este título es apenas una pequeña, ínfima metáfora del

Inferno). Allí también se menciona el amor, aunque creo que es

mejor no insistir en esa palabra, al menos por ahora.

Pero la nueva marca en el cielo, no en la cara, ese será el Paraíso.

(Fragmento encontrado entre tus ruinas).

-y terminará desarrollando su propuesta en el texto Literatura, lenguaje y sociedad (CENECA, 1983), que situaría los rasgos textuales, los gestos programáticos $\mathrm{y}$, además, el corpus de poetas que conformarían la neovanguardia, cuando ya hay algunos -varios- libros, sobre todo autoediciones, 
que lo conforman, en un mecanismo de inclusiones/exclusiones según estos propios rasgos programáticos, pero a posteriori, y con un innegable Esprit de Corps, tan propio y grato a las vanguardias "fundacionales". O sea, podríamos decir, que es un poeta en particular -Zurita- quién establece el verosímil de la que sería la neovanguardia -aunque jamás la denomina así en su propuesta-, una poesía de ruptura y resistencia, un corpus necesario y excluyente, para el nuevo campo cultural latinoamericano que adviene, tanto y a la vez, por mor de razones políticas (la política del texto) como de estética (la estética de la resistencia ciudadana).

\section{Rasgos textuales y el corpus de la neovanguardia}

Después de explayarse en asuntos sociológicos y lingüísticos, debemos consignar aquí que el texto que comentamos no tiene un mayor andamiaje teórico al respecto y solo se alude al trabajo "Vida cotidiana, cultura y política" de José Joaquín Brunner que se refiere al "excedente de poder simbólico" que le viene dado al régimen totalitario de la época por una ausencia de "ofertas competitivas".

En base a este presupuesto, Zurita construye el corpus -que, posteriormente, y a la época, presenta una densidad aún bastante líquida como para trazar un canon, digo yo afortunadamente- de los textos que fundan la neovanguardia de los años 70/80 en Chile:

"Pues bien, las obras que nítidamente -afirma- resultan excéntricas en relación al conjunto (de una muestra de 88 libros publicados y siguiendo el "método Joyceano que hace mención a los 10 libros más malos del mundo que hacen posible los otros) son las siguientes: Aguas servidas de Carlos Cociña (1981, autoedición), Luis XIV de Paulo de Jolly (1982, autoedición), La nueva novela y La poesía chilena de Juan Luis Martínez (1977 y 1978, autoediciones), Exit de Gonzalo Muñoz (1981, autoedición); Purgatorio y Anteparaíso de Raúl Zurita (malo es citarse pero peor es enmascararse) publicados por Editorial Universitaria y Editores Asociados y Fragmento de La Tirana de Diego Maquieira (autoedición, 1983). Para Zurita los textos citados "presentan un quiebre respecto a los siguientes elementos comunes al resto de los otros libros aparecidos: distanciamiento de las formas propiamente antipoéticas; Dis- tanciamiento de la poesía de los lares (Jorge Teillier es su arquetipo y a la vez su más completo exponente). Distanciamiento de la poesía epigramática (Armando Uribe es el autor que más ha seguido en esta línea). Distanciamiento de la retórica nerudiana".

De manera opuesta a la construcción de los textos antes descritos, la neovanguardia representa una visón: Fragmentaria del hablante en oposición al yo incuestionado del conjunto de las obras y una temática totalizadora frente al fragmentarismo presente del resto. $Y$ define estos constructos en 
la "relación del hablante" con disímiles y varios aspectos que caracterizarían lo que, vagamente, se ha denominado por la crítica post-vanguardias y que sería lato e inútil discutir acá, y que se puede inferir por lo ya expuesto por el cuadernillo Literatura, lenguaje y sociedad.

Aunque Zurita no cita en el texto que comentamos a la llamada Escena de Avanzada, indudablemente esta propuso y propició los signos vanguardistas que operaron como dispositivos fundamentales para recrear una "escena", análoga, pero recontextualizada, como decíamos, de las vanguardias fundacionales, en donde la incorporación de la imagen, sobre todo múltiples significantes televisivos, incluso con el aparato mismo como una suerte de simbolizante fractal, la intervención urbana, el cuerpo del artista como espacio de represión, marginación, tortura y flagelación, entraron a dirimir el estado de cosas en el campo cultural, en una suerte de torción tanto simbólica como factual hacia las insubordinaciones, para seguir la terminología de Richard, vanguardistas históricas.

Si, como se ve, la Escena de Avanzada -sinónimo de la reelaboración vanguardista en un espacio signado por la violencia sobre todo física: el cuerpo fue la primera y última víctima de la dictadura- fue en lo esencialmente plástico y visual -sonde se agruparon en torno a la experimentación más extrema desde hacía años, artistas como Carlos Leppe, Juan Dávila, Catalina Parra, etcétera, dentro del grupo CADA hubo también escritores, poetas y narradoras, como Raúl Zurita y Diamela Eltit. Richard en el texto citado también incluye en la Escena de Avanzada a poetas de la neovanguardia como Diego Maquieira, Juan Luis Martínez, Soledad Fariña y Eugenia Brito.

Así, el corpus de la llamada neovanguardia se sigue ampliando y desterritoralizando según el crítico que la estudie y los rasgos con que se la constituya. Es más, en una reciente publicación que se preocupa del problema, Maquinarias Deconstructivas. Poesía y juego en Juan Luis Martínez, Diego Maquieira y Rodrigo Lira (Santiago, 2013), Marcelo Rioseco afirma que si bien comparten algunos rasgos, no es pertinente relacionar directamente a la neovanguardia chilena con las vanguardias históricas, dado que las primeras se preocupan más por la transgresión y experimentación de los soportes -también incluye acá el textual- que por la resistencia política al régimen pinochetista. El asunto es que ya sea los estudios de Rodrigo Cánovas, Iván Carrasco, Julio Espinosa Guerra, Marcelo Ríoseco u Oscar Galindo, entre los más importantes, hay convergencias y divergencias, acuerdos y desacuerdos, diálogos y polémicas. Pero creo que, ya superados los difíciles años de la dictadura, y sean quienes sean en definitiva quienes fueron parte de llamémosle una neovanguardia orgánica e instalada, o quienes hayan transitado o la hallan prefigurado literariamente -no se puede negar el influjo de Parra: con el ya citado Quebrantahuesos; sus Artefactos -hay una relación con ellos de La Poesía Chilena de Juan Luis Martínez, aunque sea indirecta, sobre todo en el formato de caja y tarjetas que incorporan signos y significantes- o El Paseo Ahumada de Enrique Linh, y habría que indagar, rastrear y resignificar otros poemarios de la época. 


\section{Gonzalo Muñoz: apariciones, desapariciones y reapariciones. Un ejemplo.}

Gonzalo Muñoz (1956) es un poeta que establece ciertos vínculos con la llamada escena de Avanzada chilena y el grupo CADA, en particular, en los años mil novecientos ochenta, al incorporar en su escritura procedimientos visuales y performáticos. Aunque su escritura no adhiere al happening ni a la visualidad inscrita materialmente en ella, sí dialoga intertextualmente con un corpus de poetas que sí se integra con estas prácticas y otros procedimientos textuales y transgresores de la lírica acostumbrada antes de 1973. Nos referimos a una constelación de autores que por su permanente y pertinaz deseo o necesidad de ruptura y transgresión con el contexto de la época, se conoció en el decurso de nuestra lírica como neovanguarda. Creemos que los poetas y los libros que instalaron la neovanguardia en Chile fueron Juan Luis Martínez con La nueva novela (1977) y La Poesía chilena (1978) y Raúl Zurita con Purgatorio (1979) y Anteparaíso (1981) más algunos textos programáticos (el Mein Kampf de Raúl Zurita, publicado en CAL N 1, 1979 o su cuadernillo Literatura, arte y sociedad, SENECA, 1983) -a la manera de los manifiestos de la vanguardia orgánica o fundacional, cuya finalidad era, por una parte, refundar un decir rupturista y transgresor, y por otra, a través de este, crear un constructo estratégico para ingresar como una forma de resistencia artística al periodo de la dictadura pinochetista y al desgarro y la violencia cultural infringido por este; mas también vital, al que se vieron enfrentados los artistas del heterogéneo campo cultural de los años 1970/1980.

Muñoz publicó tres libros entre 1981 y 1984, Exit, Este y la Estrella negra, con una impresionante cohesión y evolución compositivas, por lo que pueden constituir una trilogía orgánica e interrelacionada entre sí. Después publicó un poema en el tríptico La Venus Negra que editó junto a los poetas de la generación que le sucedió, Guillermo Valenzuela y Víctor Hugo Díaz. Sus últimas noticias en Chile fue su inclusión en la antología de poetas hispanoamericanos Medusario. Muestra de poesía latinoamericana. Selección y notas de Roberto Echavarren, José Kozer y Jacobo Sefani, (FCE, México, 1996) junto a Diego Maquieria, Raúl Zurita y Paulo de Jolly -integrantes también de la llamada neovanguardia de las décadas citadas-. Posteriormente vino el silencio -siempre inquietante, y nunca sin un porqué, ya sea creativo o vivencial- y viajó a México en 1991. En Ciudad de México no desapareció en la tragedia del alcohol como el cónsul de Malcolm Lowry o se lo devoró Comala como a Ambroce Bierce, sino, paradójicamente, para sus contemporáneos y amigos, se dedicó a su profesión: la nada poética y muy neoliberal tarea de ejecutivo de una empresa de publicidad. ¿Una especie de posmoderna aventura rimbaudiana del silencio en el delirante país azteca del siglo XXI en lugar del África del siglo XIX?

No precisamente. Por mor a la reedición de la colección de poesía de la Universidad Diego Portales de Exit/Este, en 2010, con un evocador y lúcido prólogo del filósofo Pablo Oyarzún, las nuevas generaciones de poetas y lectores (quizá y seguramente los mismos unos y otros), tuvieron noticias de su escritura, y sus contemporáneos y amigos, que no había claudicado de la poesía. Respecto a su situación en la chilena poesía de los 80 , rememora Oyarzún en el prólogo a Este/Exit: "Al hilo de los diálogos bajo la sombra 
benéfica de un parrón, me hice la idea, seguramente peregrina, de que Zurita era la desesperación del principio (por eso de que todo principio es precipicio), Maquieira, la exasperación del final (la inversión paródica de la escatología), y Muñoz, en tanto, la errancia del signo, el ínterin de la representación, el status deviationis, la historia".

Eugenia Brito, en su análisis de la propuesta de Gonzalo Muñoz, en el texto "Literatura, Historia, revelación: La escritura poética de Gonzalo Muñoz", Campos Minados, Cuarto propio, Santiago de Chile, 1994, parte de la inscripción de su obra en el llamado neo-barroco, por primera vez en Chile: una escitura que inaugura su propuesta desde el significante, desde el juego de la intertextualidad en particular con Severo Sarduy y su novela Cobra, estableciendo su aproximación a través de las nociones barthesianas de su acercamiento a una lectura desde el placer, en una sus tres acepciones de "El placer del texto", fundamentalmente la de la escritura como goce, como placer de minar los códigos, de movilizar la escritura libidinalmente ocupando los espacios en blanco de la página con redistribución que oscila peligrosamente entre la muerte de la palabra y el espacio en blanco donde la letra tiembla, placentera, pero ostensiblemente consciente del abismo en el que se escapa de la escritura usual:

Gonzalo Muñoz se presenta a la "nueva escena literaria" como una alternativa diferente a la generada por Zurita y Maquieira. Diferente es el punto de vista de sus proposiciones estéticas y desde su peculiar modo de leer la historia de Chile y de Latinoamérica, lo que aparece vastamente documentado en Este, su segundo libro, Ed. Universitaria, Stgo, 1983 y finalmente La Estrella Negra, Francisco Zegers Editor, Santiago de Chile, 1987. Exit, su primer libro, introduce en Chile el neo-barroco de Sarduy, no ya como teoría aprendida en universidades, sino como práctica significante. Desde luego, la dedicatoria del libro a Totem, Tigre, Escorpión y Tundra, los cuatro fetiches en que se desdobla Cobra, hacen aparecer al autor y a una de sus más importantes producciones, en toda su productividad que genera este libro como actividad de pensar, pero, más allá de esa mención a su valor de intertexto, el neo-barroco desde la pluma de muñoz recupera para la poesía chilena, los juegos del significante, a partir de sus operaciones de transferencia.

En efecto, ya el segundo libro de Muñoz, Este, es una mirada también barroca ahora a la identidad Latinoamericana, pero desde la marginalidad urbana y la violencia fundacional identitaria, que introduce un grado más de adentrarse en la historia del continente en un cronotopos donde la escena fundacional es la de la conquista y la tachadura del otro. La muerte, esta vez, es presentada desde una suerte de teatralidad barroca, de una mise en scene que se prefigura ya desde la tapa del libro -en las ediciones de la época la gráfica pasaba a formar parte integral en tanto significante del libro: mutatis mutandi: Purgatorio de Raúl Zurita, La tirana de Diego Maquieira. En la La Estrella Negra ya la escritura de Muñoz se ha alejado de la estética neo-barrocca de Exit, y 
su prosa -es un largo poema en prosa sobre la marginalidad y la delincuencia "heroica" en las poblaciones durante la dictadura- sin dejar de participar en una escritura poética, se va haciendo más "transparente".

Dos sentidos, fundamentalmente, van estructurando la totalidad de este libro: lo marginal y lo épico. Hay un apígrafe al final del volumen, más allá de su propia escritura, casi confundido con un colofón que lo predetermina (o post-determina) estructuralmente: -"La estrella negra"completa un tríptico: la escena que convoca una suerte de escritura épica latinoamericana, surgida del cruce de las redes de la pasión, la política y la delincuencia". Otro epígrafe, al abrirse el libro, programa: "Gonzalo Muñoz exhuma la pasión velada de aquellos seres que se condenaron en la mitología de su desamparo". Ambos contienen los sentidos estructurantes del libro: lo marginal y lo épico.

Lo primero es un código que en la literatura chilena de los años 70/80, bajo la dictadura, irrumpió en múltiples textos a nivel de discurso en sus relaciones con el contexto (Título de Dominio de Jorge Montealegre; Lumpérica de Diamela Eltit). La palabra poética, práctica históricamente no central, bajo la unilateralidad del poder de la dictadura pinochetista, desplegó su multiplicidad de sentidos desde el margen para el margen. Por otra parte, la poesía chilena de los 70/80 pobló su escena de hechos, prácticas y sujetos cuya ubicación ha sido determinada desde la expulsión de los ejes centrales desde donde se ejerció el poder y se predeterminó la legalidad. Tenemos, en primer lugar, que La Estrella Negra, cuya autopercepción textual es la épica, se vierte a la pasión, la política y la delincuencia. Estas tres prácticas se encuentran en las redes escriturales del texto. La pasión es una energía pulsional que debe ser retenida, contenida, canalizada para el mantenimiento del orden ya sea tribal o individual; la práctica política en la 'poca de publicación del libro sufre también de una estigmatización y prohibición de la vida pública del país y la delincuencia es una constante que remnite desde nuestro mundi de hombres normales a la otredad, a lo peligroso y destructivo para "el hombre honrado considerado por él mismo" (Bataille: "Sade y el hombre normal", en El erotismo, Tusquest, Madrid, 2009).

Lo marginal llevado al extremo asume en La estrella negra la imagen del delincuente. La delincuencia, como figura, se constituye en la primera parte del libro "La gran marcha de los héroes", que se abre con el enunciado "Tenemos Caído". La figura inaugural de La estrella negra es la del caído: el texto axhumaría, develaría, traería a la superficie desde lo oculto de la pasión -en su sentido original de pathos- las formas de lo marginal en tanto derrumbamiento.

Para Bataille, la transgresión permitida del interdicto o prohibiciones sistemáticas para el tránsito hacia la humanidad, fue reducida por el cristianismo al desmoronamiento; así, la figura del rebelde se transforma en la del caído, la imagen de quien es despojado de su humanidad, reducido a la bestialidad: el demonio, las prostitutas, los delincuentes: los que saben su marginalidad y la desean: hecha la reducción, el delincuente puede ser abatido como la misma presa de una cacería sin culpa social. ("Se viene abajo como ser humano... hubo caza") 
La estrella negra configura enunciativamente en su primera parte una imagen de la marginalidad heroica en la figura del delincuente abatido sin culpa social: "Cabecilla abatido: el pistolero argentino Aníbal Israel Liverol Molina, uno de los cabecillas de la fuga de la cárcel pública ocurrida el 9 de agosto pasado y del atraco bancario de ayer, fue abatido a tiros por las fuerzas policiales". En este recorte incluido en el cuerpo del libro, en tanto la representación de la imagen del delincuente -recorte y retrato de crónica roja periodística- relaciona a La estrella negra con Eloy de Carlos Droguett, libro cada vez más actual en la narrativa chilena.

Ambos textos a través de la narratividad poemática, van metaforizando el sentido de lo marginal desde al ámbito de la delincuencia en una discursividad múltiple, que no deja de remitir a la antropología y la sociología dentro de la ficción: el delincuente, en el texto de Muñoz, es ahora una estrella que invierte su signo al trastrocar el blanco por el negro y, en este giro, "Comienza a alumbrar" sobre Santiago, una luz oscura, fantasmagórica, intersticial.

La estrella negra, como la mayoría de los textos que se instalan en la marginalidad de la delincuencia - pensamos en las novelas de Jean Genetopera por inversión de signos, pero, en este texto en particular, al contrario que en Genet, esta inversión asume formas constructivas, transformadoras, revolucionarias y socializadoras del otro: "Se trata de la lucha final por el tesoro/ El gran asalto/ Los cabecillas quieren dar el gran/ (A) Salto/ Por todos nosotros/ su caída habrá sido útil/..."

En la segunda y tercera parte: "Sombras alzadas" y "Mil hogueras", se continúa desarrollando una narratividad poética y además, el procedimiento de puesta en escena de la palabra tan propio de Muñoz, donde el lenguaje busca en un intento de visualización a través de la misma palabra, tangibilizar su discurso: lo narrativo en este libro se enuncia en el subtítulo como poesía, sería un primer nivel de relación de La estrella negra con la épica.

A nivel de sentido, en primer término se puede considerar la empresa, el interés del colectivo -aun si este es representado por una figura particular, un yo que transita hacia el nosotros y un sentido que entrega la etimología de la épica, e pepos, donde la agonía -el agon-social o individual es la energía que produce o posibilita el movimiento -la empresa. A nivel de discurso habría que considerar la introducción de la narratividad en la lírica, propia del poema épico ya sea de tradición pagana, cristiana, o hispanoamericana. La misma novela hispanoamericana, gasta mediados del siglo XX se inserta en una tradición épica -pensemos en la crónicas fundacionales y novelas fundamentales para la literatura del continente como Maladrón de Miguel Ángel Asturias se subtitula "Epopeya de los Andes Verdes". Desde este punto de vista, también deben ser considerados textos que participaron de la épica, las novelas de Alejo Carpentier, García Márquez, Roa Bastos y José María Arguedas, entre muchos otros.

Épica, marginalidad, identidad latinoamericana, el caído, la nueva estreIla, las fuerzas de la agonía, le pathos, el deseo, el transito del yo hacia el nosotros, para la construcción de lo nuevo mediante una escritura poética que participa de lo narrativo, de lo espacial de la página, de lo escénico y 
de lo denotativo: estos son, creo algunos rasgos presentes en La estrella negra, el último libro que conocimos de Gonzalo Muñoz que junto a Exit y Este cierra una trilogía notable.

Ahora bien, entrado el siglo XXI, la reedición de Exit/Este, tuvo diría yo una recepción del libro más bien pobre, casi nula. No lo reinstaló en el campo literario del actual milenio, fuera de la Universidad y también escasamente dentro de ella. Solo dos artículos -académicos- uno de Juan Herrera, "El texto en representación: La escritura visual de Gonzalo Muñoz" (Acta literaria, No 38, Concepción, Primer semestre 2009) y "Estéticas visuales como estrategias subyacentes de lo escrito: Interpretación de un poema del libro Exit de Gonzalo Muñoz" de Samir Said Soto (2014) (http://letras.s5.com. Consultado en octubre de 2014) les ha permitido, a los lectores del nuevo milenio, internarse en su proyecto escritural de los años 80 y a sus contemporáneos reevaluar su magnífica propuesta escritural. Y si el lector repara en las fechas, el texto de Herrera es anterior a la reedición de Exit/Este de las Ediciones Universidad Diego Portales y el de Said Soto, cuatro años posterior. Entre medio solo hay silencio de la crítica, y algunas menciones, al pasar, de algunos poetas más jóvenes, como Guillermo Valenzuela o Héctor Hernández Montesinos.

A propósito de la reedición citada, en The Clinic de abril de 2010, Vicente de Undurraga conversa con Muñoz en una entrevista titulada "Tras 25 años, reaparece Gonzalo Muñoz: El sexto tigre de la poesía chilena", refiriéndose al cuento de Roberto Bolaño "Encuentro con Enrique Lihn", de Putas asesinas. En este relato narra Bolaño -o el narrador ficcional, o ambos entreveradosque en una carta de Enrique Lihn el vate le habló de los que serían "los siete tigres de la poesía chilena a la época": Rodrigo Lira, Claudio Bertoni, Diego Maquieira, Juan Luis Martínez, el mismo Bolaño y Gonzalo Muñoz, que según el emisor de la carta/¿ficción?,: "se perdió en México me dijeron". (Se puede ver que por lo menos cuatro de los nombrados se inscribirían en la neovanguardia, según establezca el canon en cuestión, como veremos más adelante.

La entrevista, por supuesto oscila entre la mitificación y la desmitificación. La mitificación surge del cuento de Bolaño, canonizador por antonomasia y pulsión, y tal vez profesión, y la desmitificación de las respuestas del propio Gonzalo Muñoz. En la entrevista De Undurraga a la pregunta: "Hace ya 25 años que dejaste de publicar poesía. ¿Seguiste escribiendo?", responde, lacónicamente: "-No he dejado de escribir, quizá he cambiado radicalmente mi concepción del acto de escribir". A la siguiente pregunta en relación a qué consiste ese cambio Muñoz responde, distanciada, pero lúcidamente:

"Tiene que ver, primero, con no escribir para un lector de capilla, el poeta de la casa de enfrente o un crítico en especial, que fueron los motores de la actividad literaria de esos años en Chile. Ese sentido claustrofóbico y provinciano de la escritura es el que estando fuera- estalla en mil pedazos. Segundo, el ritmo de la publicación no es relevante para mí. En esos tiempos se calculaba cuánto tiempo se debía dejar pasar entre una publicación y otra para construir una obra. Se pensaba -por paradójico que 
fuera- en cuál era la estrategia más atinada para causar un efecto en el mercado cultural, o en los dadores de becas, o en quién hiciera falta. Al no ser ya un operador cultural ni un agitador literario, obviamente evalúas las cosas de otro modo. Menos exhibicionismo, menos show, más carne y más vida. Escribo desde la perspectiva de que casi nadie me lee. Escribo sin mucha esperanza y escribo porque es lo que mejor sé hacer. Por supuesto escribo lejos de los exhibicionismos que caracterizaron esos años, y lejos también de esa pretendida -¿ipretenciosa?- vinculación con la historia".

Sin duda han pasado 35 años en la concepción estética, literaria y vivencial en Gonzalo Muñoz, como en otros sobrevivientes, de la llamada neovanguardia de los años 80, que como en el surrealismo, se han distanciado del movimiento -si se le puede llamar como tal-, o no sobrevivieron al intento de vivir poéticamente manteniendo esa pertinaz actitud de transgresión, de ruptura y de tensión irreverente con el campo cultural en cuestión (que por lo demás ha sufrido consuderables transformaciones).

Quizá los poetas que siguen vigentes, es decir que la máquina productora de sentidos que es el texto poético, no se ha entrabado, son Carlos Cociña, y, un tanto a la sombra, un tanto de "culto" como se suele decir, Paulo de Jolly, Diego Maquieira, Bruno Vidal, que yo agregaría acá con ese gesto neovanguardista que comenzó a construirse escénicamente en los años 80 y, por supuesto Raúl Zurita. Cociña ha apostado más por una vanguardia que revisita a sus postulados de Envés, en Concepción, una vanguardia más formalista que política, más experimental que vitalista. De Jolly, para mi gusto un poeta excepcional, sería como el decadentista de la neovanguardia de los ochenta, un poeta cuyo interés pervive en la radicalidad porosa de su propuesta, de un único libro que reduce el mundo -y lo amplía a un periodo específico -y curiosísimo- de la Historia, aunque para Voltaire fue el mejor momento de la Monarquía francesa, el de Luis XIV, el Rey Sol, donde la crítica se divide en considerarlo un ex cercano al pinochetismo, un conservador a ultranza, o un neobarroco cuya potencia erótica, trasgresora y política hay que saberla leer con cautela y siguiendo su ¿juego?

Diego Maquieria y Bruno vidal sin duda son los más feroces, los más irónicos y paródicos, los más disruptivos y violentos, en suma, dos poetas cuya eficacia transgresora y cuyo talento -porque no hay otra palabra para apreciarlo- con el arte de la guerra -o guerrilla- discursiva es inigualable, sobre todo en el caso de Maquieira tan cercano a Huidobro. Zurita, sea como sea, es un poeta completo, totalizador, sin fisuras, y si las hay, son esas fisuras y desgarrones propias de toda gran poesía y un proyecto abarcador y totalizador que cumplió con creces su Main Kampf.

Considerando, como lo indica el título de esta ponencia, anuncia que vamos a hablar de una escena en "conflicto", y que si bien toda escena poética es producto o escenifica un conflicto -la del 90 por ejemplo el conflicto de la rasero neoliberal y sus consecuencias- creo que la escena de la generación de 1987 es mucho más intensa, desgarrada y problemática que 
las que la siguen, por las razones del corte republicano que hubo en el país y las consecuencias que todos acá sabemos y sería redundante o estaría ya demás especificar. Pero la pregunta es, finalmente, ¿de qué sirvió todo eso si sirvió para algo? ¿Que nos queda de esta generación si no perdida más bien NN, innominada como decía el poeta Jorge Montealegre, y, sobre todo, de la llamada neovanguardia? Porque de la poesía escrita por mujeres, de la poesía etnocultural o, simplemente étnica la respuesta me parece mucho más prístina, más incluso fundante de decires o escenas actuales, que están vigentes y bullentes hoy, a junio de 2016, y que han seguido la huella de esos, estos remotos y actuales tiempos a los que me he querido referir.

Junio de 2016 